\title{
Nanoparticles Modified by Encapsulation of Ligands with a Long Alkyl Chain to Affect Multispecific and Multimodal Imaging
}

\author{
Young Kyoung Lee ${ }^{1-3}$, Jae Min Jeong ${ }^{1-3}$, Lathika Hoigebazar ${ }^{1,3}$, Bo Yeun Yang ${ }^{1-3}$, Yun-Sang Lee ${ }^{1}$, Byung Chul Lee ${ }^{1}$, \\ Hyewon Youn ${ }^{1,4,5}$, Dong Soo Lee ${ }^{1,3,6}$, June-Key Chung ${ }^{1-3}$, and Myung Chul Lee ${ }^{1,3}$ \\ ${ }^{1}$ Department of Nuclear Medicine, Institute of Radiation Medicine, Seoul National University College of Medicine, Seoul, South \\ Korea; ${ }^{2}$ Cancer Research Institute, Seoul National University College of Medicine, Seoul, South Korea; ${ }^{3}$ Department of Radiation \\ Applied Life Science, Seoul National University College of Medicine, Seoul, South Korea; ${ }^{4}$ Institute of Radiation Medicine, Medical \\ Research Center, Cancer Research Institute, Seoul National University, Seoul, South Korea; ${ }^{5}$ Laboratory of Molecular Imaging and \\ Therapy, Cancer Research Institute, Seoul National University, Seoul, South Korea; and ${ }^{6}$ Department of Molecular Medicine and \\ Biopharmaceutical Sciences, WCU Graduate School of Convergence Science and Technology, Seoul National University, Seoul, South \\ Korea
}

The attachment of specific ligands to the surfaces of nanoparticles is important for medical and biologic imaging. However, covalent modification of nanoparticles has inherent problems in reproducibility because of many factors such as temperature, $\mathrm{pH}$, concentration, and reaction time. Thus, we developed a method for modifying nanoparticles by encapsulation with specific ligand-conjugated amphiphiles. Methods: We synthesized special amphiphiles with a hydrophilic head and a long single-alkyl chain, such as arginine-glycine-aspartic acid- $\mathrm{C}_{18}$, mannose- $\mathrm{C}_{18}$, lactose- $\mathrm{C}_{18}$, and 2-(p-isothiocyanatobenzyl)-1,4,7-triazacyclononane-1,4,7-triacetic acid- $\mathrm{C}_{18}$. And then we produced stable quantum dots (QDs) encapsulated with polysorbate 60 (a branched polyethylene glycol head with a $\mathrm{C}_{18}$ tail) and the synthesized special amphiphiles. The prepared encapsulated QDs were subject to in vitro and in vivo animal biodistribution studies and small-animal PET studies to confirm their specific binding. Results: The encapsulated QDs could specifically bind to target cells in vitro and in vivo and could be labeled with ${ }^{68} \mathrm{Ga}$ (a positron emitter) easily and with high efficiency. Conclusion: The nanoparticles encapsulated with special amphiphiles could provide a straightforward and novel imaging solution with multimodality and multispecificity.

Key Words: nanocrystal; amphiphile; Tween 60; mannose; lactose; Q-dot; RGD

J Nucl Med 2012; 53:1462-1470

DOI: 10.2967/jnumed.111.092759

$\mathbf{T}$ he specific delivery of nanoparticles to target tissues is one of the most important issues in terms of their medical

Received May 9, 2011; revision accepted Apr. 12, 2012.

For correspondence or reprints contact: Jae Min Jeong, Department of Nuclear Medicine, Seoul National University Hospital, 101 Daehangno Jongno-gu, Seoul 110-744, Republic of Korea.

E-mail: jmjng@snu.ac.kr

Published online Aug. 2, 2012.

COPYRIGHT (C 2012 by the Society of Nuclear Medicine and Molecular Imaging, Inc. applications but has proven to be difficult to achieve in practice. Nevertheless, this specificity is essential for in vivo targeting and for in vitro histologic studies or binding assays. Several researchers have described multispecific nanoparticles that bear 2 or more different types of surface ligands to enable effective agent delivery (1).

Multimodality is another important noninvasive in vivo image development issue that reflects monitoring multiple molecular signals for imaging such as fluorescence, MR, surface-enhanced Raman spectra, radioactivity, and near infrared in a rapid and quantitative manner $(2,3)$.

Generally, the biologic properties of nanoparticles are dependent on the ligands-such as biotin, antibodies, aptamers, peptides, chelating agents, fluorescent dyes, or radioisotopes-attached to their surfaces. Polyethylene glycol (PEG) molecules are also often conjugated covalently to reduce nonspecific normal-tissue uptake by a stealth function (4). However, the conjugation of ligands to nanoparticles by covalent bonding has inherent difficulties. First, the chemical reactions required to create covalent bonds are sensitive to temperature, $\mathrm{pH}$, impurities, solvents, reagent concentrations, light, time, mixing, and other factors. Furthermore, if the simultaneous conjugation of 2 or more ligands is required, the situation becomes more complicated.

To solve these problems, we designed a method of incorporating ligands to nanoparticles based on simple hydrophobic interactions between them and amphiphileshydrophilic ligands containing a long alkyl chain. Many amphiphiles possess a hydrophilic head and 1 or 2 hydrophobic tails. Those with a single head and tail are known to form micelles and to solubilize oil into water and thus are commonly used as detergents. On the other hand, amphiphiles with 1 head and 2 tails tend to form lipid bilayers or multilayers and are used to produce liposomes $(5,6)$.

In this study, we focused on the use of single-tail amphiphiles that can emulsify oil into nanosized droplets, 
but the same principle could be used to coat hydrophobic nanoparticles and facilitate their solubilization. In addition, nanoparticles can be coated with special amphiphiles with particular ligand head groups, which we refer to as specific amphiphiles (Fig. 1A). The advantage of this process is that it can introduce several different ligands by a single step with high reproducibility. The conjugation yield is not affected by the environmental conditions because the amphiphiles would be dissolved homogeneously to achieve the highest entropy. And thus all the encapsulated nanoparticles may have the homogeneous composition. This method can be applied for many hydrophobic nanoparticles, such as metals, metal oxides, carbon nanotubes, and quantum dots (QDs). Furthermore, some hydrophilic nanoparticles, such as polymers and dendrimers, can be converted to hydrophobic nanoparticles by covalently attaching hydrophobic residues to their surfaces. In the present study, we selected polysorbate 60 for coating with PEG, arginine-glycineaspartic acid (RGD) peptide for angiogenesis imaging, mannose for reticuloendothelial system imaging, and lactose for hepatocyte imaging. All these side chains are hydrophilic small molecules and thus are adequate to test our hypothesis.

\section{MATERIALS AND METHODS}

\section{General}

Organic CdSe/ZnS quantum dots (QD655 ITK and QD545 ITK) were purchased from Invitrogen. Chemical reagents were purchased from Sigma-Aldrich. To determine labeling efficiencies, instant thin-layer chromatography silica gel (ITLC-SG) purchased from Pall Corp. was used. Radioactivities on ITLCSG plates were scanned using a System 200 imaging scanner (Bioscan). Radioactivities in animal tissues were measured using a Cobra II $\gamma$-scintillation counter (Packard). A small-animal Vista scanner (GE Healthcare) was used for PET/CT. A Varioskan Flash fluorometer (Thermo Electron Corp.) and a DU 650 spectrophotometer (Beckman) were used for in vitro fluorescence detection, and a Maestro Imaging System (CRI Inc.) was used for ex vivo and in vivo fluorescence imaging. Cell fluorescence imaging was performed using a confocal laser scanning microscope (LSM 510 META; Carl Zeiss) and a fluorescence microscope (VENOXAHBT3; Olympus).

\section{Synthesis of Ligands Conjugated with Stearyl Chains}

We synthesized specific amphiphiles for coating QDs by conjugating long alkyl chains with various ligands (Supplemental Scheme 1; supplemental materials are available online only at http://jnm.snmjournals.org). Detailed synthetic procedures, yields, and spectral data are described in the supplemental information.

\section{Encapsulation of QDs with Amphiphiles}

Polysorbate 60 was the only commercially available (SigmaAldrich) amphiphile used in this study. All of the other functionalized amphiphiles (RGD- $\mathrm{C}_{18}$, lactose [Lac]- $\mathrm{C}_{18}$, mannose [Man]- $\mathrm{C}_{18}$, and 2-( $p$-isothiocyanatobenzyl)-1,4,7-triazacyclononane-1,4,7-triacetic acid [NOTA]- $\mathrm{C}_{18}$ ) were synthesized by conjugating long alkyl chains with various ligands.

After the removal of chloroform from QD655 (100 pmol) with inert gas, $4 \%(\mathrm{v} / \mathrm{v})$ polysorbate 60 solution in distilled water
(2 $\mathrm{mL}$ ) was added. The mixture was then heated to $70^{\circ} \mathrm{C}$ and then stirred in a vortex mixer overnight.

For QD545, 6\% (v/v) polysorbate 60 solution in $2 \mathrm{~mL}$ of distilled water was added to a QD545 (100 pmol) suspension in chloroform $(50 \mu \mathrm{L})$, and the 2-phase system obtained was then heated at $70^{\circ} \mathrm{C}$ for $3 \mathrm{~h}$ with intermittent stirring in a vortex mixer to produce a single-phase system. To prepare encapsulated QDs, functionalized amphiphiles $(2 \%-5 \%[\mathrm{~mol} / \mathrm{mol}]$ of polysorbate 60$)$ were added to the polysorbate 60 solution before encapsulation.

The reaction mixtures containing QD655T and QD545T were applied to a Sephacryl S-300 column (Sigma-Aldrich) $(6.7 \times 150$ $\mathrm{mm}$ ) and eluted with $10 \mathrm{mM}$ borate buffer $(\mathrm{pH} \mathrm{7.4)}$ to remove unbound polysorbate 60 and other amphiphiles. Fractions (1 mL) were collected and analyzed using a fluorometer and an ultraviolet-visible spectrometer. The encapsulated QD solutions were concentrated by ultrafiltration (Amicon Ultracel, 100-kDa cutoff; Millipore). The final concentrations of encapsulated QDs were determined by ultraviolet-visible absorbance (7).

Hydrodynamic radii and the size distributions of the purified encapsulated QDs were measured by dynamic light scattering (DLS) (Zetasizer Nano ZS90 system; Malvern Instrument Ltd.). For DLS measurements, QD solutions were diluted in distilled water and sonicated for $1 \mathrm{~min}$. Particle sizes and particle size distributions (volume-percentage value) were obtained at $25^{\circ} \mathrm{C}$ using a scattering angle of $90^{\circ}$. Transmission electron microscopy (TEM) images were taken at an acceleration voltage of 80 keV (JEM-1400; Jeol). To obtain negative-stain TEM images of encapsulated QDs, QD solutions were dropped onto a Formvar carbon-coated copper grid (SPI-Chem) and stained with saturated uranyl acetate solution.

\section{Stability Test}

The stabilities of encapsulated QDs in water were determined by storing samples at $4^{\circ} \mathrm{C}$ for a month. Sephacryl S-500 (SigmaAldrich) column $(6.7 \times 150 \mathrm{~mm})$ gel filtration was used to separate aggregated and disaggregated QDs. In addition, aggregation was also investigated using DLS measurements and by TEM observation.

\section{${ }^{68}$ Ga Radiolabeling}

QD545 coated with polysorbate 60 containing NOTA- $\mathrm{C}_{18}$ (NOTA-QD545T, $50 \mathrm{nM}, 100 \mu \mathrm{L}$ ) was dissolved in $2 \mathrm{M}$ sodium acetate buffer $(300 \mu \mathrm{L}, \mathrm{pH} 5.2)$ and incubated with ${ }^{68} \mathrm{GaCl}_{3}$ solution $(500 \mu \mathrm{L}, 300-500 \mathrm{MBq})$ for $25 \mathrm{~min}$ at $47^{\circ} \mathrm{C}$. Labeling efficiencies were measured using ITLC-SG eluted with $0.1 \mathrm{M}$ citric acid and were greater than $98 \% .{ }^{68} \mathrm{Ga}-\mathrm{NOTA}-\mathrm{QD} 545 \mathrm{~T}$ was purified using a NAP-10 column (GE Healthcare) and phosphatebuffered saline ( $\mathrm{pH}$ 7.2-7.4) for subsequent experiments. For the in vivo study, labeled solutions were concentrated using an Amicon filtration system.

The stabilities of ${ }^{68} \mathrm{Ga}-\mathrm{NOTA}-\mathrm{QD} 545 \mathrm{~T}(0.37 \mathrm{MBq})$ in $0.5 \mathrm{~mL}$ of phosphate-buffered saline at room temperature and in human serum $\left(0.5 \mathrm{~mL}\right.$ at $\left.37^{\circ} \mathrm{C}\right)$ were checked at $2 \mathrm{~h}$ after preparation. Release of free ${ }^{68} \mathrm{Ga}$ was checked by ITLC-SG at 5, 15, 30, 60, and $120 \mathrm{~min}$, and release of ${ }^{68} \mathrm{Ga}-\mathrm{NOTA}-\mathrm{C}_{18}$ was checked by Sephacryl S-500 column chromatography.

\section{Cell Culture and Animals}

The U87MG human glioma cell line was purchased from the American Type Culture Collection and grown in Eagle minimum essential medium (Gibco Laboratory) containing $10 \%(\mathrm{v} / \mathrm{v})$ heatinactivated fetal bovine serum (Gibco Laboratory) and 1\% (v/v) 


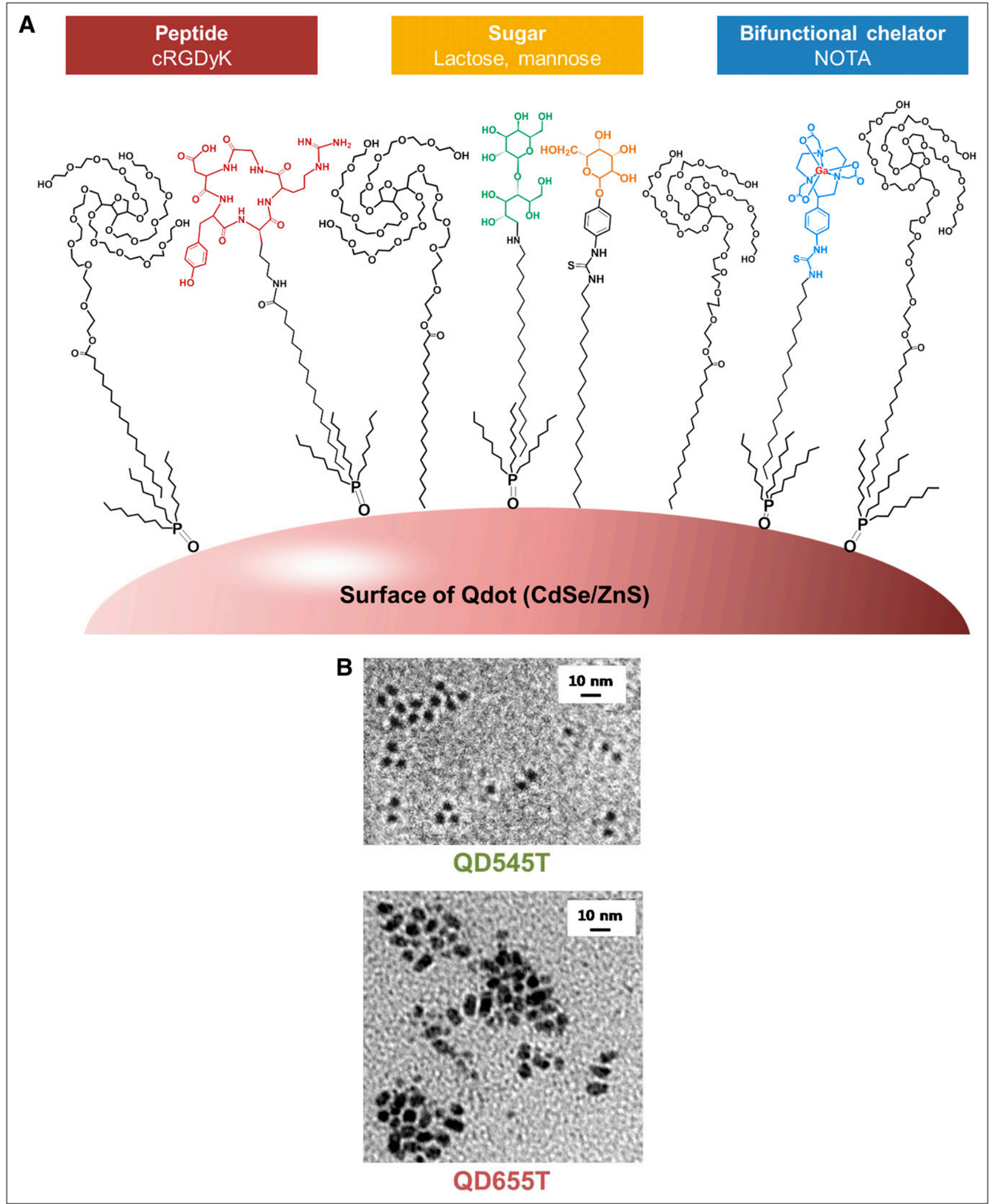

FIGURE 1. Hydrophobic QD encapsulated by amphiphiles with long alkyl tail and hydrophilic ligand head. Suitable ligands include peptides, sugars, and bifunctional chelating agents. Tails of these amphiphiles hydrophobically interact with trioctylphosphine oxide on QD surfaces. Diagram (A) and TEM images of QD545T and QD655T (B) show homogeneously suspended QDs in water after encapsulation, otherwise aggregate (negatively stained with uranyl acetate; accelerating voltage, $80 \mathrm{keV} ; \times 300,000$ ). 
antibiotics (Antibiotics \& Antimycotics [100×]; Gibco). The A431 human squamous cancer cell line was obtained from the Korean Cell Line Bank and maintained in RPMI 1640 medium (Gibco Laboratory) containing $10 \%(\mathrm{v} / \mathrm{v})$ heat-inactivated fetal bovine serum and $1 \%(\mathrm{v} / \mathrm{v})$ antibiotics at $37^{\circ} \mathrm{C}$ in a $5 \% \mathrm{CO}_{2}$ atmosphere. The MCF-7 human breast cancer cell line was also purchased from American Type Culture Collection and cultured under the same conditions as the A431 cell line.

Specific pathogen-free 4-wk-old female BALB/c nude mice and ICR mice were obtained from Orient Bio. All animal experiments were performed using a protocol approved by the Institutional Animal Care and Use Committee of the Clinical Research Institute at Seoul National University Hospital (an Association for the Assessment and Accreditation of Laboratory Animal Care, an accredited facility).

For ex vivo and in vivo studies, U87MG and A431 tumor models were established by subcutaneously injecting U87MG cells $\left(2 \times 10^{5}\right.$ cells $/ 0.1 \mathrm{~mL}$ of medium $)$ and A431 cells $\left(1 \times 10^{7}\right.$ cells $/ 0.1 \mathrm{~mL}$ of medium) into the left and right thighs of BALB/c nude mice, respectively. Tumors were excised after being grown to approximately $1 \mathrm{~cm}^{3}$.

\section{In Vitro Cell-Binding Assay}

The binding of ${ }^{68} \mathrm{Ga}-\mathrm{NOTA}-\mathrm{QD} 655 \mathrm{~T}-\mathrm{RGD}$ to U87MG, A431, and MCF-7 cells was examined in vitro. Cultured cells were harvested after trypsin (Gibco Laboratory; $1 \mathrm{~min}$ at room temperature) treatment and then washed with minimum essential medium (U87MG) or RPMI 1640 (MCF-7 and A431). Harvested cells were plated in 24 -well plates $\left(2 \times 10^{5}\right.$ cells per well $) 1 \mathrm{~d}$ before binding experiments, and the cultured cells obtained were washed sequentially with Dulbecco phosphate-buffered saline (DPBS) and with cell-binding buffer ( $20 \mathrm{mM}$ tris, $150 \mathrm{mM} \mathrm{NaCl}, 2 \mathrm{mM} \mathrm{CaCl}_{2}$, $1 \mathrm{mM} \mathrm{MnCl}_{2}, 1 \mathrm{mM} \mathrm{MgCl}_{2}$, and $0.1 \%$ [w/v] bovine serum albumin; pH 7.4). One-milliliter (0.37 MBq) aliquots of ${ }^{68} \mathrm{Ga}-N O T A-$ QD655T-RGD or ${ }^{68} \mathrm{Ga}-N O T A-Q D 655 T$ in cell-binding buffer were added to each well in triplicate and incubated at $37^{\circ} \mathrm{C}$ under $5 \% \mathrm{CO}_{2}$. A blocking study was performed to determine the nonspecific binding of ${ }^{68} \mathrm{Ga}-N O T A-Q D 655 T-R G D$ in the presence of cyclic Arg-Gly-Asp-D-Tyr-Lys (cRGDyK) $(10 \mu \mathrm{M})$. The cancer cells were incubated at $37^{\circ} \mathrm{C}$ for $15 \mathrm{~min}$, washed with chilled DPBS 3 times, and suspended in 1\% sodium dodecyl sulfate solution $(0.5 \mathrm{~mL} /$ well). Radioactivities were measured using a $\gamma$ counter. Cellular protein in each well was measured using BCA protein assay kits (Pierce).

\section{Imaging of In Vitro Cell Binding}

For confocal imaging, $1.5 \times 10^{4}$ cells were plated per chamber (8-well glass chamber slides, Lab-Tek Chamber Slide System; Nalge Nunc International) and grown for $16 \mathrm{~h}$. The cells were washed with warmed cell-binding buffer solution and incubated with QD655TRGD (50 nM) or QD655T (50 nM) in $200 \mu \mathrm{L}$ of cell-binding buffer solutions for $15 \mathrm{~min}$. The cell-binding buffer was then aspirated from each chamber, and cells were washed carefully 3 times with chilled DPBS. They were then fixed with $3.7 \%$ paraformaldehyde solution and mounted using 4'-6-diamidino-2-phenylindole (DAPI) solution to stain nuclei. Confocal imaging was performed using a laser scanning microscope equipped with a 488-nm laser for QD655 and a 405-nm laser for DAPI and QD545.

\section{Ex Vivo Tumor Tissue Staining}

Tumor tissues obtained from U87MG-xenografted BALB/c nude mice were cryosectioned at $-20^{\circ} \mathrm{C}$ at $7 \mu \mathrm{m}$, fixed in cold acetone $\left(-20^{\circ} \mathrm{C}, 20 \mathrm{~min}\right)$, incubated with $10 \%$ (v/v) fetal bovine serum (room temperature, $30 \mathrm{~min}$ ), and then incubated with QD655T-RGD (30 nM, $100 \mu \mathrm{L})$ at $4{ }^{\circ} \mathrm{C}$ overnight. Blocking studies were performed using a U87MG tissue slide pretreated with free cRGDyK (500 nM, $100 \mu \mathrm{L})$ for $1 \mathrm{~min}$. For confocal microscopy, treated tissue slices were mounted and counterstained with DAPI.

\section{In Vivo Fluorescence Imaging}

QD655T-RGD (120 nM, $100 \mu \mathrm{L})$ or QD655T (127 nM, $100 \mu \mathrm{L})$ was injected into U87MG and A431 tumor-xenografted mice via a tail vein. Organs were excised at $1 \mathrm{~h}$ after injection and imaged using a Maestro imaging system equipped with a yellow filter set (excitation, 575-605 nm; emission, $645 \mathrm{~nm}$ ). Excised tumors in optimal cutting temperature medium (Tissue-Tek O.C.T. compound; Sakura Finetek) were sectioned while frozen $(7 \mu \mathrm{m})$, fixed with $3.7 \%(\mathrm{v} / \mathrm{v})$ paraformaldehyde solution, and mounted with DAPI solution for confocal microscopy.

\section{In Vivo PET}

${ }^{68} \mathrm{Ga}-\mathrm{NOTA}-\mathrm{QD} 655 \mathrm{~T}-\mathrm{RGD}(30-40 \mathrm{MBq}$ in $0.1 \mathrm{~mL}$ of phosphate-buffered saline) prepared as described above was injected into U87MG and A431 tumor-xenografted mice via a tail vein. For the blocking study, cRGDyK ( $1 \mathrm{mM}, 0.1 \mathrm{~mL})$ was coinjected. Dynamic whole-body PET scans were obtained over $10 \mathrm{~min}$ in 20 frames $(10 \times 60 \mathrm{~s})$. The images were obtained by 3 -dimensional Fourier rebinning using a 2-dimensional ordered-subsets expectation maximization reconstruction algorithm with scatter, decay, and attenuation correction from raw framed sonograms. For each PET scan, 3-dimensional regions of interest were drawn over tumors and major organs on whole-body axial images. Standardized uptake values were obtained using MMWKS-Vista software from reconstructed data. Animal PET/CT was performed at $1 \mathrm{~h}$ after injection under isoflurane anesthesia.

\section{Ex Vivo Confocal Microscopy Imaging of Livers and In Vivo PET}

Normal ICR mice (age, $6 \mathrm{wk}$ ) were sacrificed at $20 \mathrm{~min}$ after lactose-conjugated QD545T (QD545T-Lac, $100 \mathrm{nM}, 160 \mu \mathrm{L}$ ) and mannose-conjugated QD655T (QD655T-Man, $100 \mathrm{nM}, 40 \mu \mathrm{L}$ ) were coinjected via a tail vein. Livers were excised immediately, frozen in OCT compound at $-20^{\circ} \mathrm{C}$, and sectioned at $7 \mu \mathrm{m}$ using a cryotome. Sections were fixed in $3.7 \%$ paraformaldehyde solution for $20 \mathrm{~min}$, stained, and mounted using DAPI mounting solution. The localizations of QD545T-Lac and QD655T-Man in the liver sections were observed by confocal microscopy. For PET, ${ }^{68} \mathrm{Ga}$-NOTA-QD655T-Man (25.5 MBq/0.1 mL) and ${ }^{68} \mathrm{Ga}-N O T A-$ QD545T-Lac (37.8 MBq/0.1 mL) were prepared as described above and injected through a tail vein into normal ICR mice. Animals were imaged under isoflurane anesthesia 10 min later.

\section{RESULTS}

\section{Encapsulation of QDs Using Polysorbate 60 and Specific Amphiphiles}

Initially, we confirmed that polysorbate 60 could encapsulate hydrophobic QDs and dissolve them in water. QD655 or QD545 was incubated in $4 \%$ or $6 \%$ aqueous polysorbate 60 , respectively, and then dissolved in water. Other ligands were introduced by simply adding specific amphiphiles $(2 \%-5 \%[\mathrm{~mol} / \mathrm{mol}]$ of polysorbate 60$)$, such as RGD-C ${ }_{18}$, Man- $\mathrm{C}_{18}$, Lac-C$_{18}$, and NOTA-C 18 , into polysorbate 60 solutions before QD encapsulation, and as a result, various encapsulated QDs were produced (Table 1). 
TABLE 1

Encapsulated QDs Produced Using Various Additional Amphiphiles

\begin{tabular}{|c|c|c|}
\hline Added amphiphile & QD545 & QD655 \\
\hline None & QD545T & QD655T \\
\hline $\mathrm{RGD} \mathrm{C}_{18}$ & QD545T-RGD & QD655T-RGD \\
\hline Lac-C $_{18}$ & QD545T-Lac & QD655T-Lac \\
\hline Man- $\mathrm{C}_{18}$ & QD545T-Man & QD655T-Man \\
\hline NOTA-C $_{18}$ & NOTA-QD545T & NOTA-QD655T \\
\hline NOTA-C ${ }_{18}$ and RGD-C 18 & NOTA-QD545T-RGD & NOTA-QD655T-RGD \\
\hline NOTA-C ${ }_{18}$ and Lac-C 18 & NOTA-QD545T-Lac & NOTA-QD655T-Lac \\
\hline NOTA-C ${ }_{18}$ and Man- $C_{18}$ & NOTA-QD-545T-Man & NOTA-QD655T-Man \\
\hline
\end{tabular}

The schematic formation of water-soluble QDs coated with various specific amphiphiles is shown in Figure 1A. These encapsulated QDs were purified using Sephacryl S-300 as previously described (Supplemental Fig. 1) $(8,9)$. The hydrodynamic size of QD545T determined by DLS was $9.5 \pm 1.3 \mathrm{~nm}$. However, the hydrodynamic size of QD655T could not be determined by this method because of fluorescence emission. The particle diameters of QD545T and QD655T determined by TEM were $9.3 \pm$ 0.6 and $15.7 \pm 1.8 \mathrm{~nm}$, respectively (Fig. 1B). The fluorescence spectra showed a narrow peak at $650 \pm 2.85 \mathrm{~nm}$ for QD655 and QD655T and a peak at $549 \pm 2.65 \mathrm{~nm}$ for QD545 and QD545T, representing the fact that the fluorescence spectra of neither QD is affected by encapsulation. The stabilities of encapsulated QDs in water were determined by size-exclusion gel chromatography (Sephacryl S-500), DLS, and TEM and found that they were stable in water and formed no aggregate after more than 4 wk of storage (Supplemental Fig. 2).

For quantitative study, NOTA-conjugated QDs were labeled with ${ }^{68} \mathrm{Ga}$, and labeling efficiencies were analyzed by thin-layer chromatography. For this analysis, ITLC-SG was eluted with $0.1 \mathrm{M}$ citric acid solution. Free ${ }^{68} \mathrm{Ga}$ moved with the citric acid solution solvent front $\left(\mathrm{R}_{\mathrm{f}}=1.0\right)$, and ${ }^{68} \mathrm{Ga}$-labeled QDs remained at the origin $\left(\mathrm{R}_{\mathrm{f}}=0.0\right)$. The labeling efficiency of NOTA-conjugated QDs with ${ }^{68} \mathrm{Ga}$ was greater than $98 \%(n>4)$, and no free ${ }^{68} \mathrm{Ga}$ was found after purification (radiochemical purity $>99 \%, n>4$ ) using a Sephadex G25 column (Sigma-Aldrich). Absence of dissociation of ${ }^{68} \mathrm{Ga}-\mathrm{NOTA}-\mathrm{C}_{18}$ from nanoparticles was confirmed by Sephacryl S-500 chromatography (Supplemental Fig. 3C). ${ }^{68} \mathrm{Ga}$-labeled QD derivatives were stable for more than $2 \mathrm{~h}$ at room temperature $(>97 \%)$ in prepared medium and at $37^{\circ} \mathrm{C}$ in human serum $(>96 \%)$, which is enough for practical purposes considering the short half-life (68 min) of ${ }^{68} \mathrm{Ga}$ (Supplemental Fig. 3).

\section{Cell-Binding Test of RGD-Conjugated QDs}

To confirm the specific binding of RGD- $\mathrm{C}_{18}$-encapsulated QDs to $\alpha_{\mathrm{v}} \beta_{3}$ integrin-expressing cells, QD655 was encapsulated with $4 \%$ polysorbate 60 solution containing NOTA-C ${ }_{18}(2 \%[\mathrm{~mol} / \mathrm{mol}]$ of polysorbate 60$)$ or RGD-C ${ }_{18}$ $(5 \%[\mathrm{~mol} / \mathrm{mol}]$ of polysorbate 60$)$ and then labeled with ${ }^{68} \mathrm{Ga}$. U87MG cells (a human glioma cell line expressing a high level of $\alpha_{\mathrm{v}} \beta_{3}$ integrin), MCF-7 cells (a human breast cancer cell line expressing a low level of $\alpha_{\mathrm{v}} \beta_{3}$ integrin), and A431 cells (a human squamous cancer cell line expressing a low level of $\alpha_{\mathrm{v}} \beta_{3}$ integrin) were investigated with respect to ${ }^{68} \mathrm{Ga}-N O T A-Q D 655 T-R G D$ and ${ }^{68} \mathrm{Ga}-$ NOTA-QD655T binding in vitro. ${ }^{68} \mathrm{Ga}-\mathrm{NOTA}-\mathrm{QD} 655 \mathrm{~T}-$ RGD bound to U87MG, and this binding was blocked by the presence of free cRGDyK $(10 \mu \mathrm{M})$ and also showed nonspecific binding to the other cells (Fig. 2A). However, ${ }^{68} \mathrm{Ga}-\mathrm{NOTA}-\mathrm{QD} 655 \mathrm{~T}$ showed only nonspecific binding to the 3 cell lines examined. The considerable amount of nonspecific binding is regarded as endocytotic uptake. However, the binding of ${ }^{68} \mathrm{Ga}-N O T A-Q D 655 T-R G D$ was significantly higher than nonspecific binding $(P<0.05)$.

QD655T-RGD was also used as a fluorescence probe for the detection of $\alpha_{v} \beta_{3}$ integrin on cell surfaces by confocal microscopy (Fig. 2B). Cell nuclei were stained with DAPI. The results were consistent with a cell-binding assay performed with ${ }^{68} \mathrm{Ga}$-labeled QDs. High uptake was achieved only by the incubation of U87MG cells with QD655T-RGD and was blocked by the coincubation with $100 \mu \mathrm{M}$ of cRGDyK. MCF-7 and A431 cells showed only slight uptake when they were incubated with either QD655T or QD655T-RGD.

\section{Ex and In Vivo Angiogenesis Targeting Using RGD-Conjugated QDs}

We performed an ex vivo study to examine the specific binding of QD655T-RGD to $\alpha_{\mathrm{v}} \beta_{3}$ integrin-positive U87MG cancer tissue. About $1 \mathrm{~cm}^{3}$ of U87MG cancer tissue samples was obtained from xenografted nude mice, cryosectioned at $7 \mu \mathrm{m}$, and incubated with QD655T-RGD solution overnight. Strong red fluorescence by QD655 was observed, especially at tumor peripheries (Supplemental Fig. 4A). However, preincubation of U87MG cancer tissue with free cRGDyK (500 nM) blocked the binding of QD655T-RGD to tissue, demonstrating its specific binding nature (Supplemental Fig. 4B). Furthermore, QD655T did not show any binding to the cancer tissues, indicating that QDs encapsulated by only polysorbate 60 cannot bind to $\alpha_{\mathrm{v}} \beta_{3}$ integrin-expressing tumors.

The specific delivery of encapsulated QDs to $\alpha_{v} \beta_{3}$ integrin-expressing tumor was investigated using an in vivo fluorescence distribution experiment and an animal PET 


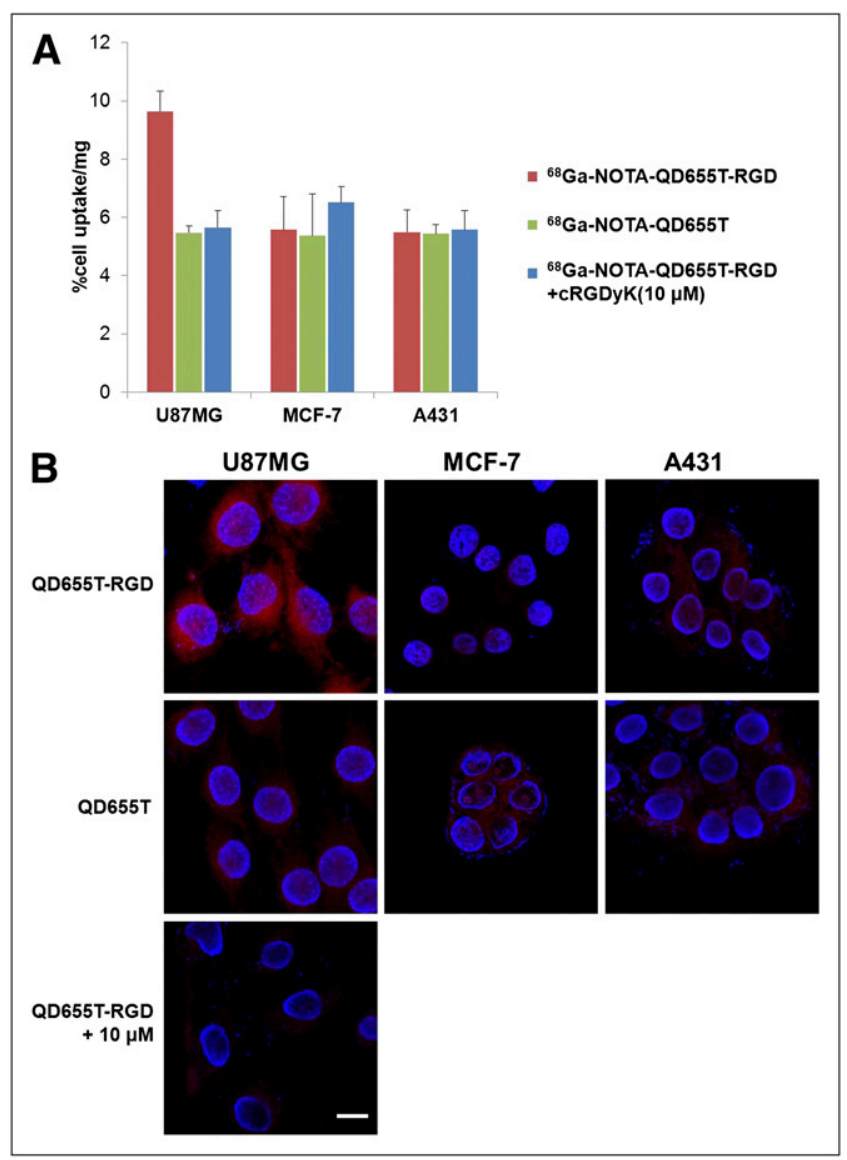

FIGURE 2. Cellular uptake of QD655T-RGD. (A) In vitro cell-binding assay. Only U87MG incubated with ${ }^{68} \mathrm{Ga}-\mathrm{NOTA}-\mathrm{QD} 655 \mathrm{~T}-\mathrm{RGD}$ shows significantly higher uptake than any other combinations. High uptake is effectively blocked by addition of cRGDyK $(10 \mu \mathrm{M})$. Data represent mean $\pm \mathrm{SD}(n=4)$. (B) Confocal microscopy shows consistent results with in vitro cell-binding assay. Only U87MG incubated with QD655T-RGD shows high uptake (red), which is blocked by $100 \mu \mathrm{M}$ cRGDyK. Blue fluorescence represents nuclei by DAPI staining. Scale bar indicates $20 \mu \mathrm{m}$.

study. Aliquots of QD655T-RGD (120 nM; $100 \mu \mathrm{L})$ and QD655T $(127 \mathrm{nM} ; 100 \mu \mathrm{L})$ were administered to mice xenografted with U87MG on the left thigh and A431 cancer cells on the right thigh through a tail vein. Mouse organs were excised at $1 \mathrm{~h}$ after injection, and fluorescence images were taken using the Maestro imaging system (Supplemental Fig. 5A). Despite the encapsulation of QD655 with polysorbate 60 (the PEG in polysorbate 60 reduces reticuloendothelial uptake), both polysorbate 60 and polysorbate 60 containing RGD- $\mathrm{C}_{18}$-encapsulated QDs showed somewhat high liver and spleen uptakes, indicative of reticuloendothelial system uptake. Higher accumulations of QD655T-RGD were observed in U87MG tumor masses than in A431 tumor masses and other organs. However, QD655T did not accumulate in U87MG tumors as expected. Confocal microscopy of frozen tissue sections demonstrated strong fluorescence only at the peripheries of U87MG tumors injected with QD655T-RGD, suggest- ing its specific uptake by tumor cells (Supplemental Fig. 5B).

To evaluate in vivo tumor-targeting effects, animal PET of nude mice bearing U87MG and A431 tumors was performed after the injection of ${ }^{68} \mathrm{Ga}-\mathrm{NOTA}-\mathrm{QD} 655 \mathrm{~T}$ RGD. The ${ }^{68} \mathrm{Ga}$-labeling yield in this case was greater than $98 \%$, and the amount of ${ }^{68} \mathrm{Ga}-\mathrm{NOTA}-\mathrm{QD} 655 \mathrm{~T}-\mathrm{RGD}$ injected was approximately 5 pmol. Because PET is more sensitive than fluorescence imaging, a lesser amount of ${ }^{68} \mathrm{Ga}$-labeled QD was required to identify U87MG tumor uptake (Fig. 3). ${ }^{68}$ Ga-NOTA-QD655T-RGD uptake was higher in U87MG tumors than in A431 tumors, and the U87MG tumor uptake was blocked by the coinjection of cRGDyK, demonstrating the specific uptake of ${ }^{68} \mathrm{Ga}-\mathrm{NOTA}-\mathrm{QD} 655 \mathrm{~T}-\mathrm{RGD}$. High liver uptake is supposed to indicate reticuloendothelial uptake. Standardized uptake values were calculated from PET images for quantitation purposes. The U87MG tumor-to-muscle ratio and the U87MG tumor-to-A431 tumor ratios for ${ }^{68} \mathrm{Ga}$-NOTA-QD655T-RGD were 3.5:1 and 5.5:1, respectively, representing the specific uptake to U87MG tumor.

The results showed that QDs encapsulated with RGD$\mathrm{C}_{18}$ was bound specifically to $\alpha_{\mathrm{v}} \beta_{3}$ integrin-expressing cells and tissues in vitro and in vivo, and we confirmed that these QDs could be used as bimodality probes-radiotracer and fluorescence.

\section{Targeting of Liver and Spleen Using Mannose- and Lactose-Conjugated QDs}

${ }^{68} \mathrm{Ga}-\mathrm{NOTA}-\mathrm{QD} 545 \mathrm{~T}-\mathrm{Lac}$ and ${ }^{68} \mathrm{Ga}-\mathrm{NOTA}-\mathrm{QD} 655 \mathrm{~T}-$ Man were administered to normal ICR mice through a tail vein. PET showed that both agents accumulated in the liver within 10 min of administration (Figs. 4A and 4B). Furthermore, ${ }^{68} \mathrm{Ga}$-NOTA-QD655T-Man showed high spleen uptake. Confocal fluorescence laser microscopy of the livers showed that the 2 agents had different fluorescence distribution patterns (Fig. 4C). ${ }^{68} \mathrm{Ga}$-NOTA-QD545T-Lac was diffusely taken up by hepatocytes, and its uptake reflected the structural organization of the whole liver; for example, fluorescence was concentrated at the cells surrounding the central vein holes and sinusoids. However, no uptake was found in the spleen, which contains no hepatocytes.

On the other hand, ${ }^{68} \mathrm{Ga}$-NOTA-QD655T-Man was taken up locally both in the liver and in the spleen, representing Kupffer cell uptake (Fig. 4C). Furthermore, high-contrast 3dimensional PET images revealed that ${ }^{68}$ Ga-NOTA-QD655TMan was taken up by bone marrow (Supplemental Video 1).

\section{DISCUSSION}

In the present study, we used QDs to investigate our hypothesis. Although no QD has been approved for in vivo human use, other nanoparticles, such as iron oxides, have been approved for clinical investigations, and the results of the present study can be applied to these nanoparticles. QDs have many outstanding properties as potential fluorescence sensors, such as photostability, high extinction coefficients, 

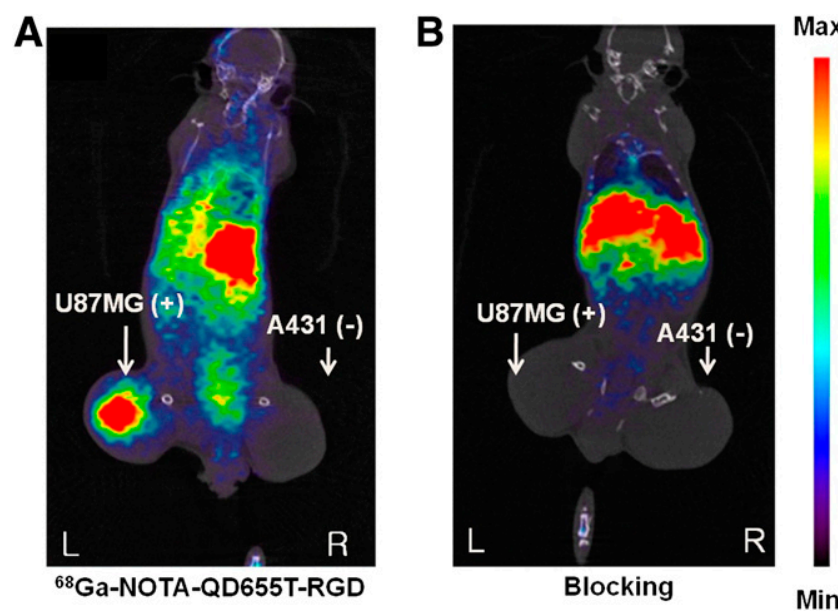

FIGURE 3. PET images (coregistered with corresponding CT images) of U87MG and A431 tumor-bearing nude mice acquired at $60 \mathrm{~min}$ after intravenous injection. (A) ${ }^{68} \mathrm{Ga}-\mathrm{NOTA}-\mathrm{QD} 655 \mathrm{~T}-$ RGD shows high uptake in U87MG tumor but not in A431 tumor. (B) ${ }^{68}$ Ga-NOTA-QD655T-RGD can be completely blocked by coinjection of free cRGDyK, representing specific uptake. Max = maximum; $\min =$ minimum.

broad absorption spectra, narrow adjustable emission spectra (from 500 to $>1,000 \mathrm{~nm}$ ), and large absorption cross-sections $(7,10)$.

High-quality QDs can be obtained by a high-temperature growth procedure using organic solvents and trioctylphosphine or trioctylphosphine oxide (11). However, QDs obtained using this procedure are insoluble in water, and thus, surface modification with hydrophilic ligands is required to dissolve them in water and to make them biocompatible. This modification could be achieved by the cap-exchange chelate effect from the mono- or dithiol groups $(1,12,13)$ or by the encapsulation onto the trioctylphosphine/trioctylphosphine oxide of the organic particles by hydrophobic absorption with silica shell (14), amphiphilic polymers (15-17), or lipids (18). The materials used to coat QDs facilitate their dissolution in water, protect QD surfaces from the aqueous environment, and offer accessible sites for chemical or biologic carriers.

The use of QDs in the biologic and clinical fields requires that they be made water-soluble and not be targeted by the immune system - that is, have stealth properties, which could be achieved by pegylation. There are various commercialized detergents having a PEG head group and a single alkyl tail such as Brij78 and polysorbate 60 . It has been reported that a trial to produce single-QD-containing micelles using Brij78 failed (18), but the same trial using polysorbate 60 succeeded (19). Thus, we used polysorbate 60 , which has a branched PEG head and a $\mathrm{C}_{18}$ stearate tail. This branching means that the polysorbate series has a larger head diameter than the Brij series. Therefore, the polysorbate series allow the encapsulation of nanosized materials more easily than the Brij series. Furthermore, the polysorbate series already are approved for clinical use (listed in various monographs such as the U.S. Pharma- copoeia, the British Pharmacopoeia, and the European Pharmacopoeia), and products are commercially available.

To provide QD compatibility with another modality, we used ${ }^{68} \mathrm{Ga}$, which is applied as a label for PET. ${ }^{68} \mathrm{Ga}$ is being increasingly used because it is readily available from a ${ }^{68} \mathrm{Ge} /{ }^{68} \mathrm{Ga}$ generator $(20-23)$.

Various specific amphiphiles were synthesized for coating QDs by conjugating a long alkyl chain with various ligands. Initially, cRGDyK peptide was conjugated with stearic acid to produce stearyl-cRGDyK (RGD-C 18 ). RGD derivatives have been used to investigate $\alpha_{v} \beta_{3}$ integrin expression in vascular and tumor angiogenesis by biologic and clinical researchers (24-29). In addition, a mannose and stearylamine conjugate (Man- $\mathrm{C}_{18}$ ) was synthesized to target the mannose receptor of Kupffer cells (30-33), and a lactose and stearylamine conjugate $\left(\right.$ Lac- $\left.\mathrm{C}_{18}\right)$ was synthesized to target the asialoglycoprotein receptor in the liver $(32,34,35)$. For radiolabeling with ${ }^{68} \mathrm{Ga}$, we conjugated the bifunctional chelating agent SCN-Bz-NOTA to stearylamine (NOTA-C ${ }_{18}$ ). A high labeling efficiency for ${ }^{68} \mathrm{Ga}$ was obtained, as expected, because NOTA is a strong chelating agent especially for gallium (36).

Specific binding of the QDs encapsulated with RGD-C 18 was proved by in vitro cell-binding and tissue-binding studies using $\alpha_{v} \beta_{3}$ integrin-positive (U87MG) and -negative (MCF-7 and A431) cell lines. The specific bindings were significantly higher than nonspecific bindings and were blocked by the addition of excessive ligands. An in vivo imaging study using U87MG- and A431-xenografted nude mice also confirmed the specific bindings. There was a report that an RGD-labeled ultrasmall superparamagnetic iron oxide similar in size to the QDs accumulated in the vasculature of xenografted U87MG tumors and this accumulation also was blocked by the coinjection of free RGD (37). However, the fact that the extent of blocking was less for the unconjugated form of ultrasmall superparamagnetic iron oxide than for the RGD-labeled form is a controversial aspect of this research. The injected cold RGD amount in the literature was $0.5 \mathrm{mg}$, but in our experiment we used 10 $\mathrm{mg}$, which could be one of the explanations for the controversy.

Man- $\mathrm{C}_{18}-$ and Lac- $\mathrm{C}_{18}$-enacpsulated QDs were tested in vivo in mice. Mannose receptor is known to exist on the surfaces of macrophages, and thus, mannosylated human serum albumin is used to target bone marrow and liver, especially Kupffer cells. On the other hand, lactosylated human serum albumin is used for liver imaging because it binds to asialoglycoprotein receptors on hepatocytes $(32,38)$. This experiment also proved specific uptake of the encapsulated QDs, representing the feasibility of wide application of the agents.

\section{CONCLUSION}

The described encapsulated QDs using amphiphiles with a long single-alkyl tail and a specially designed hydrophilic head were proved to be excellent multimodal and multi- 

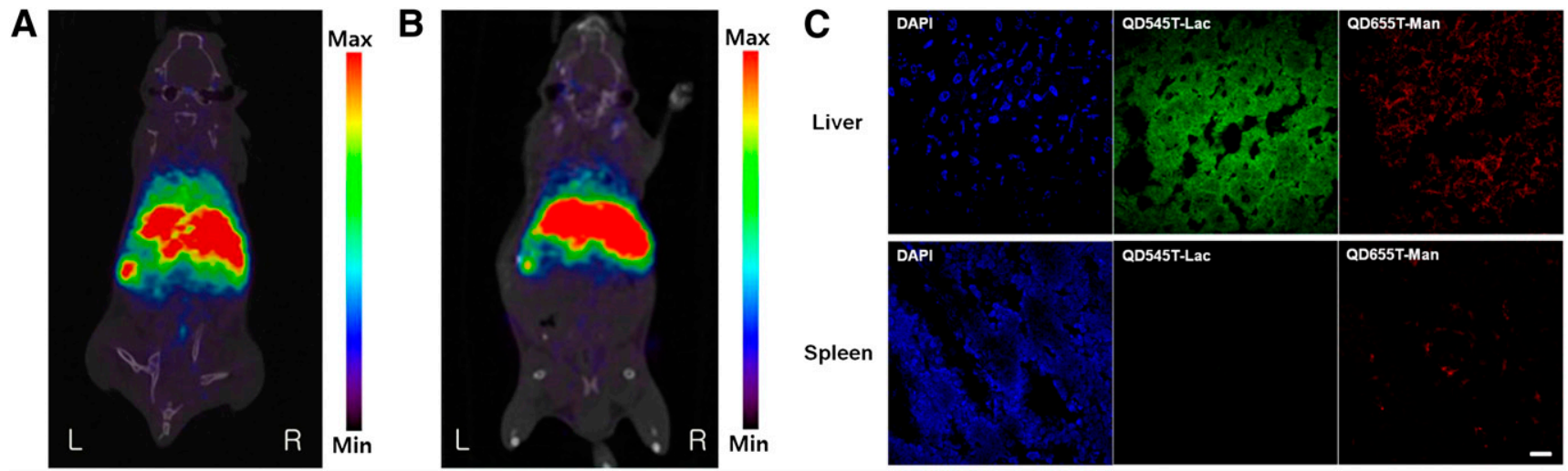

FIGURE 4. Investigation of liver and spleen distribution of QDs encapsulated with polysorbate 60 including Man- $\mathrm{C}_{18}$ or Lac- $\mathrm{C}_{18}$. PET/CT coronal images of normal ICR mice acquired at $30 \mathrm{~min}$ after injection of ${ }^{68} \mathrm{Ga}-\mathrm{NOTA}-\mathrm{QD} 655 \mathrm{~T}-\mathrm{Man}$ (A) or ${ }^{68} \mathrm{Ga}-\mathrm{NOTA}-\mathrm{QD} 545 \mathrm{~T}-\mathrm{Lac}$ (B)

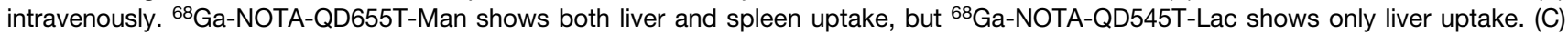
Confocal images of frozen liver and spleen slices $(7 \mu \mathrm{m})$ taken after injection of QD655T-Man (red) and QD545T-Lac (green) intravenously. Hepatocyte uptake of QD545T-Lac is seen in liver but not in spleen. QD655T-Man is taken up by reticuloendothelial system of liver and spleen. Scale bar represents $20 \mu \mathrm{m}$. Max = maximum; $\min =$ minimum.

specific nanoparticles for in vitro and in vivo targeting. For example, polysorbate 60 (an amphiphile with a branched PEG head) enables the solubilization of hydrophobic QDs and also provides the pegylation effect. In the same manner, amphiphiles with an RGD, mannose, or lactose head provide binding to $\alpha_{\mathrm{v}} \beta_{3}$ integrin, macrophages, and asialoglycoprotein receptor, respectively, and amphiphiles with a NOTA head can provide chelating ability with ${ }^{68} \mathrm{Ga}$. Furthermore, the described method is applicable to other hydrophobic nanoparticles composed of, for example, metals, metal oxides, polymers, or carbon nanotubes, and thus, the method has a great potential as a tool for molecular imaging in many fields of study.

\section{DISCLOSURE STATEMENT}

The costs of publication of this article were defrayed in part by the payment of page charges. Therefore, and solely to indicate this fact, this article is hereby marked "advertisement" in accordance with 18 USC section 1734.

\section{ACKNOWLEDGMENTS}

This research was partly supported by the Converging Research Center Program (2010K001055) and National Research Laboratory Program (R0A-2008-000-20116-0) through the Ministry of Education, Sciences and Technology. No other potential conflict of interest relevant to this article was reported.

\section{REFERENCES}

1. Susumu K, Mei BC, Mattoussi H. Multifunctional ligands based on dihydrolipoic acid and polyethylene glycol to promote biocompatibility of quantum dots. Nat Protoc. 2009;4:424-436.

2. Massoud TF, Gambhir SS. Molecular imaging in living subjects: seeing fundamental biological processes in a new light. Genes Dev. 2003;17:545-580.

3. Pathak P, Katiyar VK. Multi-functional nanoparticles and their role in cancer drug delivery: a review. J Nanotechnol Online. 2007. Available at: http://www. azonano.com/article.aspx?ArticleID=1903. Accessed July 30, 2012.

4. Akerman ME, Chan WC, Laakkonen P, Bhatia SN, Ruoslahti E. Nanocrystal targeting in vivo. Proc Natl Acad Sci USA. 2002;99:12617-12621.
5. Gruner SM. Materials. In: Ostro MJ, ed. Properties of Liposomal Bilayers in Liposomes from Biophysics to Therapeutics. New York, NY: Marcel Dekker; 1987:1-38.

6. Cressman S, Dobson I, Lee JB, Tam YY, Cullis PR. Synthesis of a labeled RGDlipid, its incorporation into liposomal nanoparticles, and their trafficking in cultured endothelial cells. Bioconjug Chem. 2009;20:1404-1411.

7. Leatherdale CA, Woo WK, Mikulec FV, Bawendi MG. On the absorption cross section of CdSe nanocrystal quantum dots. J Phys Chem B. 2002;106:7619-7622.

8. Carion O, Mahler B, Pons T, Dubertret B. Synthesis, encapsulation, purification and coupling of single quantum dots in phospholipid micelles for their use in cellular and in vivo imaging. Nat Protoc. 2007;2:2383-2390.

9. Dixit SK, Goicochea NL, Daniel MC, et al. Quantum dot encapsulation in viral capsids. Nano Lett. 2006;6:1993-1999.

10. Reiss P, Protiere M, Li L. Core/Shell semiconductor nanocrystals. Small. 2009;5:154-168.

11. Murray CB, Norris DJ, Bawendi MG. Synthesis and characterization of nearly monodisperse Cde $(\mathrm{E}=\mathrm{S}, \mathrm{Se}, \mathrm{Te})$ semiconductor nanocrystallites. J Am Chem Soc. $1993 ; 115: 8706-8715$.

12. Uyeda HT, Medintz IL, Jaiswal JK, Simon SM, Mattoussi H. Synthesis of compact multidentate ligands to prepare stable hydrophilic quantum dot fluorophores. J Am Chem Soc. 2005;127:3870-3878.

13. Dubois F, Mahler B, Dubertret B, Doris E, Mioskowski C. A versatile strategy for quantum dot ligand exchange. J Am Chem Soc. 2007;129:482-483.

14. Gerion D, Pinaud F, Williams SC, et al. Synthesis and properties of biocompatible water-soluble silica-coated CdSe/ZnS semiconductor quantum dots. $J$ Phys Chem B. 2001;105:8861-8871.

15. Yu WW, Chang E, Falkner JC, et al. Forming biocompatible and nonaggregated nanocrystals in water using amphiphilic polymers. J Am Chem Soc. 2007; 129:2871-2879.

16. Fernandez-Argüelles MT, Yakovlev A, Sperling RA, et al. Synthesis and characterization of polymer-coated quantum dots with integrated acceptor dyes as FRET-based nanoprobes. Nano Lett. 2007;7:2613-2617.

17. Wang $\mathrm{CH}$, Hsu YS, Peng CA. Quantum dots encapsulated with amphiphilic alginate as bioprobe for fast screening anti-dengue virus agents. Biosens Bioelectron. 2008;24:1018-1025.

18. Dubertret B, Skourides P, Norris DJ, Noireaux V, Brivanlou AH, Libchaber A. In vivo imaging of quantum dots encapsulated in phospholipid micelles. Science. 2002;298:1759-1762.

19. Wu H, Zhu H, Zhuang J, Yang S, Liu C, Cao YC. Water-soluble nanocrystals through dual-interaction ligands. Angew Chem Int Ed Engl. 2008;47:3730-3734.

20. Ehrhardt GJ, Welch MJ. A new germanium-63/gallium-68 generator. $\mathrm{J} \mathrm{Nucl}$ Med. 1978;19:925-929.

21. Zhernosekov KP, Filosofov DV, Baum RP, et al. Processing of generator-produced ${ }^{68} \mathrm{Ga}$ for medical application. $J$ Nucl Med. 2007;48:1741-1748.

22. Jeong JM, Kim YJ, Lee YS, Lee DS, Chung JK, Lee MC. Radiolabeling of NOTA and DOTA with positron emitting ${ }^{68} \mathrm{Ga}$ and investigation of in vitro properties. Nucl Med Mol Imaging. 2009;43:330-336.

23. Dinesh S, Lee LS, Jeong JM. ${ }^{68} \mathrm{Ga}$-labeled radiopharmaceuticals for positron emission tomography. Nucl Med Mol Imaging. 2010;44:233-240. 
24. Schottelius M, Laufer B, Kessler H, Wester HJ. Ligands for mapping alphavbeta3-integrin expression in vivo. Acc Chem Res. 2009;42:969-980.

25. Jeong JM, Hong MK, Chang YS, et al. Preparation of a promising angiogenesis PET imaging agent: ${ }^{68} \mathrm{Ga}$-labeled $\mathrm{c}(\mathrm{RGDyK})$-isothiocyanatobenzyl-1,4,7-triazacyclononane-1,4,7-triacetic acid and feasibility studies in mice. J Nucl Med. 2008;49:830-836.

26. Cai W, Chen K, Li ZB, Gambhir SS, Chen X. Dual-function probe for PET and near-infrared fluorescence imaging of tumor vasculature. J Nucl Med. 2007; 48:1862-1870.

27. Cai W, Chen X. Preparation of peptide-conjugated quantum dots for tumor vasculature-targeted imaging. Nat Protoc. 2008;3:89-96.

28. Choi HS, Liu W, Liu F, et al. Design considerations for tumour-targeted nanoparticles. Nat Nanotechnol. 2010;5:42-47.

29. Temming K, Schiffelers RM, Molema G, Kok RJ. RGD-based strategies for selective delivery of therapeutics and imaging agents to the tumour vasculature. Drug Resist Updat. 2005;8:381-402.

30. Magnusson S, Berg T. Extremely rapid endocytosis mediated by the mannose receptor of sinusoidal endothelial rat liver cells. Biochem J. 1989;257:651656.

31. Jeong JM, Hong MK, Kim YJ, et al. Development of ${ }^{99 m}$ Tc-neomannosyl human serum albumin $\left({ }^{99 \mathrm{~m}} \mathrm{Tc}-\mathrm{MSA}\right)$ as a novel receptor binding agent for sentinel lymph node imaging. Nucl Med Commun. 2004;25:1211-1217.
32. Kim S, Jeong JM, Hong MK, et al. Differential receptor targeting of liver cells using ${ }^{99 m}$ Tc-neoglycosylated human serum albumins. Arch Pharm Res. 2008; 31:60-66.

33. Choi JY, Jeong JM, Yoo BC, et al. Development of ${ }^{68} \mathrm{Ga}$-labeled mannosylated human serum albumin (MSA) as a lymph node imaging agent for positron emission tomography. Nucl Med Biol. 2011;38:371-379.

34. Krohn KA, Vera DR, Steffen SM. Neogalactoalbumin-Tc-99: a general-model for some bifunctional carbohydrates. J Label Compd Radiopharm. 1981;18:9193.

35. Jeong JM, Hong MK, Lee J, et al. ${ }^{99 \mathrm{~m}} \mathrm{Tc}$-neolactosylated human serum albumin for imaging the hepatic asialoglycoprotein receptor. Bioconjug Chem. 2004; 15:850-855.

36. Parker D. Tumor targeting with radiolabelled macrocycle-antibody conjugates. Chem Soc Rev. 1990;19:271-291.

37. Kiessling F, Huppert J, Zhang C, et al. RGD-labeled USPIO inhibits adhesion and endocytotic activity of alphav beta3-integrin-expressing glioma cells and only accumulates in the vascular tumor compartment. Radiology. 2009;253: 462-469.

38. Ma P, Liu S, Huang YB, Chen XS, Zhang LP, Jing XB. Lactose mediated livertargeting effect observed by ex vivo imaging technology. Biomaterials. 2010;31: 2646-2654. 